\title{
Response to "Comments on Abrupt-Interface Solution for Carbon Dioxide Injection into Porous Media by Dentz and Tartakovsky (2008)" by Lu et al.
}

\author{
Marco Dentz • Daniel M. Tartakovsky
}

Received: 10 February 2009 / Accepted: 11 February 2009 / Published online: 5 March 2009

(C) The Author(s) 2009. This article is published with open access at Springerlink.com

In their Comment, the authors raise an important issue of the validity of the analytical (Dentz and Tartakovsky 2008) and semi-analytical (Nordbotten et al. 2005) solutions describing migration of supercritical $\mathrm{CO}_{2}$ that is being injected into a deep geological formation. Indeed, any mathematical model (including the two-phase immiscible flow model employed in the Comment) is an abstraction, whose fidelity to a physical phenomenon it purports to describe can be established only through observation. Unlike standard multi-phase flow models, which rely on a number of implicit and often unstated physical conjectures whose validity is hard to ascertain a priori (e.g., Adler and Brenner1988; Gray and Hassanizadeh 1998, among others), the solutions of Dentz and Tartakovsky (2008) and Nordbotten et al. (2005) are based on the following premise:

1. An interfacial region between the injected $\mathrm{CO}_{2}$ and a host fluid can be approximated by an abrupt interface.

Dentz and Tartakovsky (2008) also postulate that

2. A quasi-steady (successive steady-states) description of the dynamics of this interface is adequate and

3. The Dupuit assumption of horizontal flow is applicable.

Section 2 of Dentz and Tartakovsky (2008) provides a detailed discussion of the conditions under which assumptions 1-3 are appropriate. Both the abrupt-interface approximation and the Dupuit approximation have a long history in modeling of flow and transport in porous media, including subsurface hydrogeology. The reader familiar with textbooks on the subject (e.g., Bear 1972) would find objectionable the Comment's assertion that the abrupt-interface approximation is "unphysical for immiscible displacements in porous

\footnotetext{
M. Dentz

Department of Geotechnical Engineering and Geosciences, Technical University of Catalonia (UPC), Barcelona, Spain

D. M. Tartakovsky $(\bowtie)$

Department of Mechanical and Aerospace Engineering, University of California,

San Diego, La Jolla, CA, USA

e-mail:dmt@ucsd.edu
} 
media" (see also Tartakovsky and Winter 2001; Neufeld and Huppert 2001; and many other studies that employed this approximation). Likewise, the Comment is off base in claiming that "the coexistence of two fluid phases with different densities directly contradicts the Dupuit assumption." This approximation is ubiquitous in analyses of seawater intrusion, which treat saltwater and fresh water as two distinct fluids separated by an abrupt interface (see the specific references to Bear 1972 in Dentz and Tartakovsky 2008). Finally, it is worthwhile recognizing that the validity and breakdown of the field-scale, macroscopic assumptions 1-3 are easier to verify and monitor in the field than the validity and breakdown of their pore-scale counterparts underpinning multiphase flow equations.

Rather than employing assumptions 2 and 3, Nordbotten et al. (2005) postulate that the injected mass of $\mathrm{CO}_{2}$ advances in a way that minimizes energy (work required to inject $\mathrm{CO}_{2}$ and potential energy). Our analysis of a two-dimensional injection regime (see Sect. 4.3.2 in Dentz and Tartakovsky 2008) revealed that their energy optimization approach predicts a vertical interface, i.e., plug flow. This differs, both quantitatively and qualitatively, from the expected behavior due to buoyancy effects: $\mathrm{CO}_{2}$ fronts advance further into an aquifer's top than its bottom. (Our solution captures this phenomenon.) It is in this context that we have called a prediction based on the energy-minimization postulate unphysical.

The discussion above should make it clear that the two analytical approaches are different in their foundational premises. The solution of Nordbotten et al. (2005) holds in viscosity-dominated flow regimes, while our solution is valid for buoyancy-dominated injection conditions. In this sense, the two solutions are complementary. The validity of both solutions in their respective ranges of applicability has been confirmed by the numerical simulations shown in Fig. 2 of the Comment. With this in mind, it should come as no surprise that "the two analytical solutions do not agree with one another even though both are based on the abrupt interface assumption" (see the Comment's "conclusion remark" [sic]).

Equally self-evident is the second "conclusion remark," "neither analytical solution can predict the shapes and spreading distances of displacement fronts with acceptable accuracy over a range of injection rates using more realistic capillary properties." First, both Nordbotten et al. (2005) and Dentz and Tartakovsky (2008) are explicit in describing flow regimes (injection rates, etc.) which can be described with their respective solutions. For example, Dentz and Tartakovsky (2008) state,

While an abrupt interface between immiscible (or miscible) fluids cannot exist in a macroscopic (Darcian) sense, in many situations of practical interest the transition zone between two (miscible) fluids is relatively narrow or the (immiscible) displacement is almost complete so that the approximation of an abrupt interface separating the two fluids is justified (Bear 1972, p. 439). The abrupt-interface approximation is generally valid for flow regimes with the large Péclet number, a condition that is expected to hold during $\mathrm{CO}_{2}$ injection.

The simulations reported in the Comment (Fig. 2) confirm this point.

Second, the "realistic capillary properties" are highly elusive when it comes to multiphase flows. It is especially so for subsurface flows of $\mathrm{CO}_{2}$. As far as fundamental physics is concerned, the linear, VGM, EXP, or cubic relative permeability models used in the simulations reported in the Comment are no more realistic than the abrupt-interface model.

The reliance on large numerical codes to model complex nonlinear phenomena in the subsurface is itself problematic. When used to model displacement fronts associated with $\mathrm{CO}_{2}$ injection, multiphase-flow codes (including PFLOTRAN) are known to yield results that depend on a spatio-temporal discretization of governing equations, suffer from numerical dispersion, etc. (see the Comment). Such numerical artifacts compromise the reliability 
of numerical results, which must be validated via comparison with appropriate analytical solutions, such as the one proposed by Dentz and Tartakovsky (2008).

Open Access This article is distributed under the terms of the Creative Commons Attribution Noncommercial License which permits any noncommercial use, distribution, and reproduction in any medium, provided the original author(s) and source are credited.

\section{References}

Adler, P.M., Brenner, H.: Multiphase flow in porous media. Ann. Rev. Fluid Mech. 20, 35-59 (1988)

Bear, J.: Dynamics of fluids porous media. Elsevier, New York (1972)

Dentz, M., Tartakovsky, D.M.: Abrupt-interface solution for carbon-dioxide injection in porous media. Transp. Porous Med. (2008). doi:10.1007/s11242-008-9268-y

Gray, W.G., Hassanizadeh, S.M.: Macroscale continuum mechanics for multiphase porous-media flow including phases, interfaces, common lines and common points. Adv. Water Res. 27, 261-281 (1998)

Neufeld, J.A., Huppert, H.E.: Modelling carbon dioxide sequestration in layered strata. J. Fluid Mech. in press, (2009)

Nordbotten, J.M., Celia, M.A., Bachu, S.: Injection and storage of $\mathrm{CO}_{2}$ in deep saline aquifers: analytical solution for $\mathrm{CO}_{2}$ plume evolution during injection. Transp. Porous Med. 58, 339-360 (2005)

Tartakovsky, D.M., Winter, C.L.: Dynamics of free surfaces in random porous media. SIAM J. Appl. Math. 61, 1857-1876 (2001) 